\title{
INVASIVE APPROACHES IN BENIGN BREAST TUMORS IN BRAZIL
}

Karla O. Elesbão1, Lucas C. Silva1', Mateus G. R. Silva1', Felipe Z. Pereira, Beliza O. Almeida', Lucas G. Cardoso', Fabiane A. Carvalho'

${ }^{1}$ Centro Universitário de Anápolis - UniEVANGÉLICA - Anápolis (GO), Brazil.

Objectives: Quantify the number of invasive approaches in benign breast tumor in women in Brazil, based on the variables of surgical procedures, clinical size and detection of the injury. Methodology: An ecological study, population-based and cross-sectional design. Considered the total number of invasive approaches in benign breast tumor in Brazil between 2009 and 2015. Data were collected from the Breast Cancer Information System (SISMAMA/SUS). Initially, descriptive statistics were performed and then the data were analyzed by ratio scale and relative frequency. Results: A total of 79,074 surgical procedures were performed in benign breast tumor in Brazil during the quoted period. Of these, $14.6 \%$ segmental resections and $3.8 \%$ mastectomies. Being $67 \%$ of the segmental resections and $58.4 \%$ of the mastectomies performed in tumors smaller than $5 \mathrm{~cm}$. Among the procedures, $33.8 \%$ were in fibroadenomas, with $6.7 \%$ of segmental resections and $1 \%$ of mastectomies. Being $81.8 \%$ of the segmental resections and $61.8 \%$ of the mastectomies performed in fibroadenomas smaller than $5 \mathrm{~cm}$. The detection occurred by image in $43.6 \%$ of these tumors. Conclusion: This study verified a progressive increase of invasive approaches in benign breast tumors in women between the period from 2009 to 2015 in Brazil. Among the benign tumors, $7.7 \%$ of the fibroadenomas were submitted to invasive surgical procedures, the majority of them smaller than $5 \mathrm{~cm}$. However, the literature recommends non-invasive procedures such as clinical follow-up and tumorectomy by mammotome or cryoablation. A significant number of fibroadenomas were identified by image, a high accuracy diagnostic method according to the literature. Taking into account that invasive surgical procedures are not recommended for the treatment of benign tumors, as well as the physical and emotional impacts on women's lives submitted to them, studies are relevant to understand the reasons for this approach. 